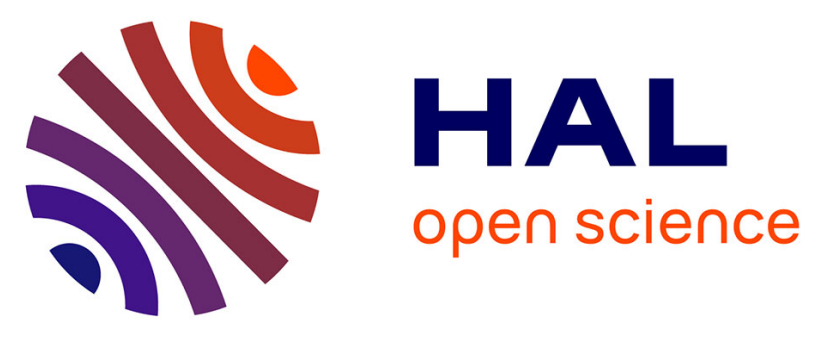

\title{
Preliminary Study on the Impact of Place and Route Strategy on FPGA Electromagnetic Emission
}

Estevan Lara, Allan Constante, Juliano Benfica, Fabian Vargas, Alexandre

Boyer, Sonia Ben Dhia, Andreas Gleinser, Gunter Winkler, Bernd

Deutschmann

\section{To cite this version:}

Estevan Lara, Allan Constante, Juliano Benfica, Fabian Vargas, Alexandre Boyer, et al.. Preliminary Study on the Impact of Place and Route Strategy on FPGA Electromagnetic Emission. 2021 Argentine Conference on Electronics (CAE2021), Mar 2021, Bahia Blanca (Virtual), Argentina. 10.1109/CAE51562.2021.9397567 . hal-03206144

\section{HAL Id: hal-03206144 \\ https://hal.laas.fr/hal-03206144}

Submitted on 23 Apr 2021

HAL is a multi-disciplinary open access archive for the deposit and dissemination of scientific research documents, whether they are published or not. The documents may come from teaching and research institutions in France or abroad, or from public or private research centers.
L'archive ouverte pluridisciplinaire HAL, est destinée au dépôt et à la diffusion de documents scientifiques de niveau recherche, publiés ou non, émanant des établissements d'enseignement et de recherche français ou étrangers, des laboratoires publics ou privés. 


\title{
Preliminary Study on the Impact of Place and Route Strategy on FPGA Electromagnetic Emission
}

\author{
Estevan L. Lara ${ }^{1}$, Allan A. Constante ${ }^{1}$, Juliano Benfica ${ }^{1}$, Fabian Vargas $^{1}$, Alexandre Boyer ${ }^{2}$, Sonia B. Dhia ${ }^{2}$, Andreas \\ Gleinser ${ }^{3}$, Gunter Winkler ${ }^{3}$, Bernd Deutschmann ${ }^{3}$ \\ ${ }^{1}$ Catholic University - PUCRS, Brazil, vargas@computer.org \\ ${ }^{2} L A A S-C N R S$, France, sonia.bendhia@insa-toulouse.fr \\ ${ }^{3}$ Institute of Electronics, Graz University of Technology, Austria, bernd.deutschmann@tugraz.at \\ Invited Paper
}

\begin{abstract}
Dense integration and high operating frequencies associated with increased number of $I / O$ buffers of FPGAs are factors contributing to electromagnetic emission (EME) increase. Consequently the radiation issue that used to be discussed at PCB level has shifted to component level. Thus it is of high interest and challenging to investigate chip electromagnetic performance. This paper presents a preliminary analysis about the impact of the placement and routing $(P \& R)$ process of logic inside the FPGA on the chip EME level. With this purpose, a softcore processor was placed and routed based on three different strategies in the configurable logic block (CLB) array of a commercial FPGA and executed an application code running over an operating system (OS). Three experiments based on farand near-field emission measurements have been performed.-The obtained results indicate that the EME level can be affected up to $\mathbf{2 1 . 8 \%}$ by the way the processor is placed and routed inside the FPGA.
\end{abstract}

Keywords - Commercial Field-Programmable Gate Array (FPGA), Reliability, Logic place and route process, Electromagnetic emission (EME), GTEM Cell Test Method, Surface Scan Test Method.

\section{INTRODUCTION}

As semiconductor technologies have been shrinking, the speed of circuits, integration density, and the number of $\mathrm{I} / \mathrm{O}$ interfaces have been significantly increased. As consequence, electromagnetic emission (EME) became a paramount issue in the integrated circuit (IC) design. In this scenario, engineers must guarantee the ability of electronic components to operate safely in an increasingly hostile electromagnetic environment. Therefore, dealing to better understand the relationship between ICs and EME, several studies have been found in the literature dealing to define measurement procedures and standards, laboratory setup and countermeasures to understand and minimize EME from ICs. King Lee Chua at al. [1] presented an alternative approach to measure electromagnetic emissions by locating the entire FPGA test board inside the GTEM cell instead of mounting it at the wall of the cell. In [2], authors evaluate and analyze the impact of I/O switching activities on the EME of a Xilinx Spartan-3 FPGA. In [3], Mohamed Ramdani at al. provide a non-exhaustive review of the research work conducted in the field of electromagnetic compatibility (EMC) at the IC level over the past 40 years with focus on measurement methods and modeling approaches. In [4], Jan Mocha et al. presented an approach that compared the conducted and radiated EME generated by two processor systems implemented in a FPGA. The same program was used in both processor systems. However, one of the systems was build using only internal resources of the FPGA, while the other used external SRAM as the program memory. In [5], authors presented and discussed the results of testing EME generated by circuits implemented in Xilinx FPGAs XC 4025E and XCV 800 types. Both radiated and conducted emissions of the circuits were measured as a function of clock frequency. At the end of this work, authors presented possible practical recommendations to minimize EME level. In [6] and [7], authors developed a customized platform tailored for combined tests of total ionizing dose (TID) radiation and electromagnetic interference (EMI). This platform is dedicated to Xilinx Spartan and Virtex FPGAs. In the sequence, they validated the platform by analyzing the single-event upset (SEU) sensitivity of a SRAM-based FPGA to the combined effects of EMI and TID-imprinted values.

Nevertheless, from the best of our knowledge it is worth noting that none of the previous works found in the literature was dedicated to study the effect of logic place and route (P\&R) on the EME level of FPGA. Dealing to minimize this gap, this paper analyzes the impact of the P\&R process of logic inside the FPGA on the emission level of such component. Though, a softcore version of the processor Hellfire RISC [8,9] was placed and routed in three different ways in a commercial FPGA (Xilinx/Spartan 3E, part number $\mathrm{XC3S500E-4PQ208).} \mathrm{In} \mathrm{order} \mathrm{to} \mathrm{measure} \mathrm{EME} \mathrm{of} \mathrm{such}$ embedded system, three experiments were performed. The first experiment measured the far-field emission from voltage raw measurements at GTEM cell terminal [10]. The second experiment was based on measuring the near magnetic field using a fixed $\mathrm{H}$-field probe within a small anechoic chamber as shielded housing to minimize ambient noise. Then, the last experiment was based on the so-called Surface Scan Method [11]. This measurement technique enables the visualization of the near magnetic field directly at the surface of an IC package or microchip. In this case an $\mathrm{H}$-field probe is automatically 
moved step by step over the surface of the IC. At each position, the magnetic field is measured with the help of e.g. a spectrum analyzer in a certain frequency position or range.

The remainder of this work is organized as follows: Section II describes the performed experiments, proceedings and laboratory set-up. Section III presents the case-study based on the Hellfire RISC softcore processor, application code and P\&R strategies for the FPGA. Then, Section IV discusses the obtained results and suggests further directions of the research. Finally, Section V draws the final conclusions of the work.

\section{PERFORMED EXPERIMENTS}

In order to observe the effect of the place and route (P\&R) algorithm on the FPGA emission level, three experiments were performed as described in the sequence.

\section{A. GTEM Cell Test Method}

The first experiment, based on the GTEM Cell Test Method $[10,14,15]$, was performed at the LAAS-CNRS and is depicted in Fig. 1. The FPGA board was placed within the GTEM cell in a fixed position. Its activity was controlled by a Test Host Computer (THC) placed outside the GTEM cell. The voltage induced at the GTEM cell terminal by the FPGA radiation was measured with a spectrum analyzer. If the FPGA board is assumed to be electrically small and equivalent to an elementary electric dipole, the far-field emission from the FPGA board can be estimated at any distance (in this experiment, at $1 \mathrm{~m}$ ) [12]. In spite of these assumptions, the results can be compared with typical radiated emission requirements at electronic equipment level.

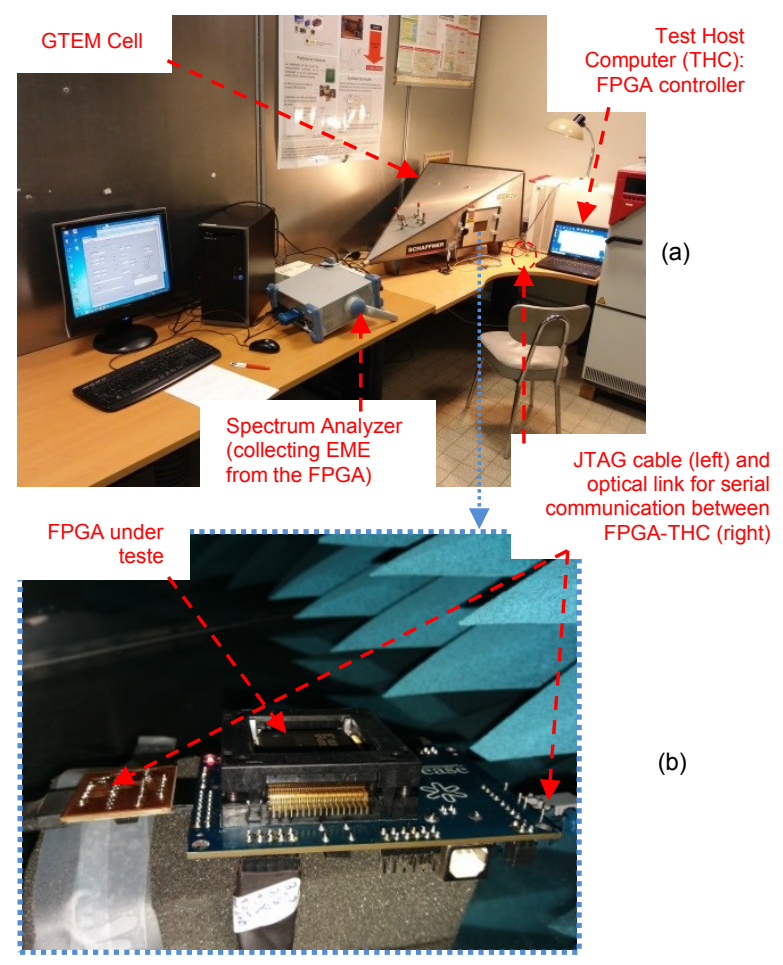

Fig. 1. GTEM Cell Test Method experiment set-up at LAAS-CNRS: (a) general overview; (b) detail of the FPGA under test.
The GTEM cell primarily estimates the far-field emission of the FPGA including the emission generated by the PCB on which the IC is mounted on. In contrast to that, the two near field measurement techniques (Sections $B$ and $C$ described hereafter) have been chosen to have a good comparison between far and near field of the generated emission by measuring only the silicon die contributions. For the experiment described in Section $B$, a big near field probe was located in a fixed center position on top of the IC package to measure the magnetic field contribution of the whole die. Unlike to this, in the last experiment (Section C) the focus was set to localize the near magnetic field distribution over the die surface by using a very small, moving near field probe, which offered a very high local resolution. These methods constitute effective methodologies to characterize integrated circuits in terms of EME.

\section{B. Anechoic Chamber with Fixed Near-Field Probe}

The second experiment, based on a near-H field probe located within a small anechoic chamber which was used as shielded housing to minimize ambient noise, was performed at the TU Graz University (see Fig. 2). The H-field probe was placed approximately $2 \mathrm{~mm}$ above the surface of the ICs package. For all measurements the probe was at the same position. The output voltage of the near-field probe was measured using a spectrum analyzer.



Fig. 2. Anechoic chamber with near-field probe experiment set-up at TU Graz: (a) general overview; (b) and (c) details of the FPGA under test.

\section{Surface Scan Method with a Moving Near-Field Probe}

The third experiment, based on the Surface Scan Method $[11,13,16]$, was also performed at the TU Graz University (Fig. 3). This method constitutes an effective methodology to characterize printed circuit boards and integrated circuits in terms of electromagnetic emission (and immunity as well). It is a useful technique to locate areas of critical radiation or 
susceptibility, which could influence the performance of devices nearby or the device under test itself. Fig. 3 depicts a photo of the test set-up. The equipment seen in this figure consists of a micromanipulation (wafer probing) that is equipped with stepper motors to control the probe movement in $\mathrm{XYZ}$ directions (in our experiment, $\mathrm{Z}$ was fixed to a constant value of $2 \mathrm{~mm}$ ). Note that while the aforementioned experiment measured emission based on a fixed probe position with respect to the FPGA package, this experiment scanned 4,000 points above the surface of the package with a moving near-field probe where the chip is located, hence adding a much higher precision and sensitivity to the emission measurement.

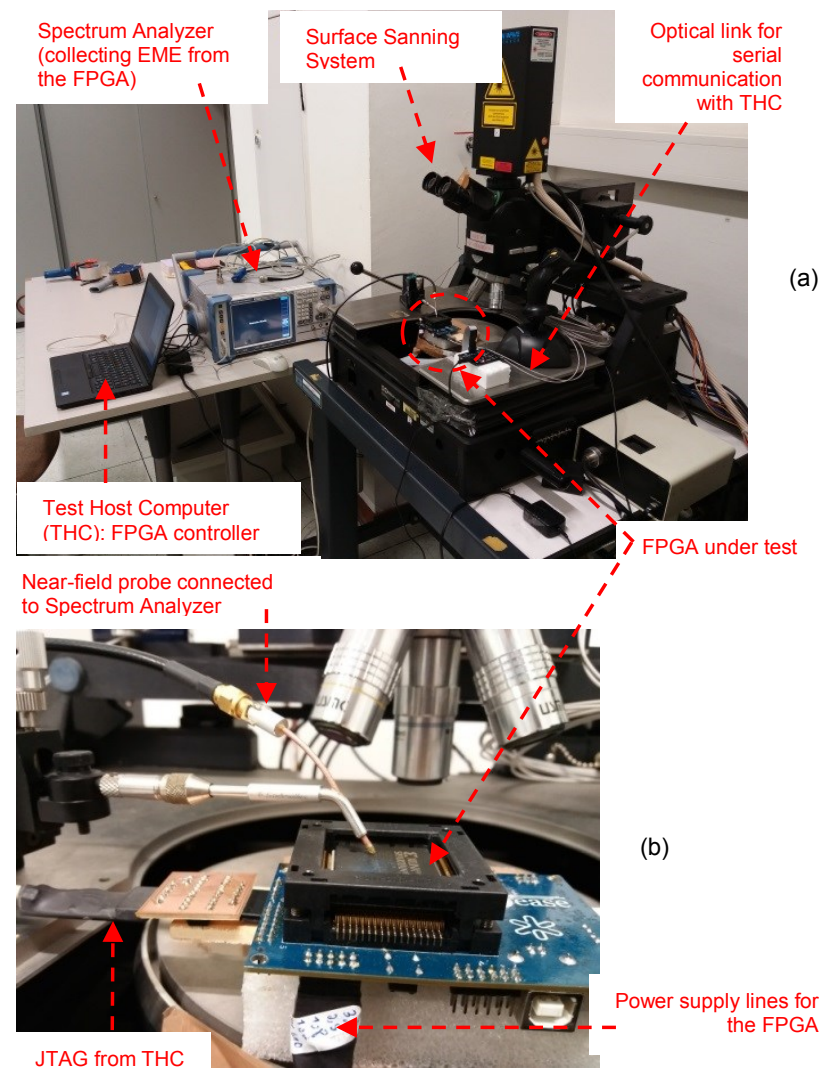

Fig. 3. Surface Scan Method experiment set-up at TU Graz: (a) general overview; (b) detail of the FPGA under test.

\section{Case-Study: HW, SW And Place \& Route STRATEGIES}

The case-study was based on a Spartan 3E FPGA (part number XC3S500E-4PQ208), from Xilinx, which was mounted on a dedicated board specially designed to support EMC test [6]. The board design and software development were carried out by the Catholic University - PUCRS. In order to analyze the effect of the P\&R algorithm on the FPGA emission level, a softcore processor: Hellfire RISC running a Bubble-Sort program over an operating system (HF-OS) $[8,9]$ was placed and routed in three different configurations in the Spartan 3E FPGA. In the first configuration, the processor was placed and routed automatically by the ISE-EDK Design Tool of Xilinx without designer assistance (let us say this is the "Automated" P\&R version). In the second and third configurations, the processor was completely and intentionally sat in the periphery region ("Center" version) and in the lefthand side ("Right" version) of the configurable logic block (CLB) array of the FPGA. With this purpose, the designer prevented the ISE-EDK Tool from instantiating the processor core in the center of the CLB array (Center version) and in the right side of the CLB array (Right version), respectively. Fig. 4 depicts these three P\&R strategies. See also Fig. 5, which depicts the embedded system basic block diagram.

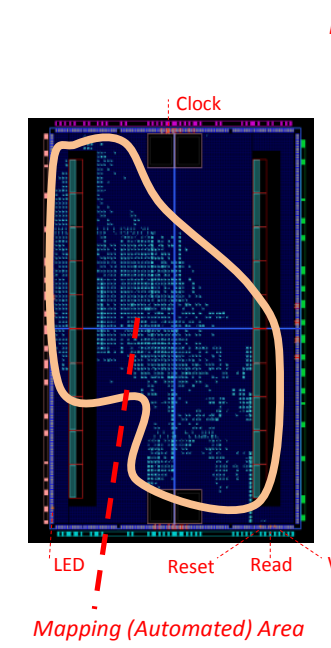

(a)



(b)

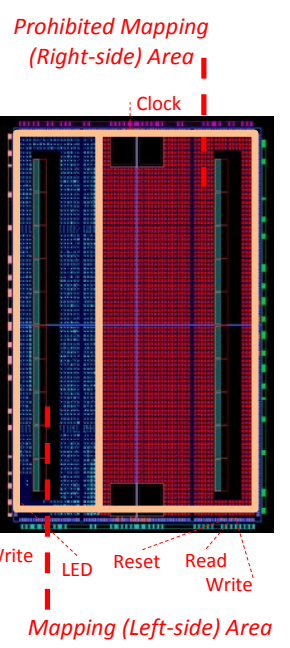

(c)
Fig. 4. Three P\&R strategies to instantiate the processor core inside the CLB array of the FPGA: (a) "Automated", (b) "Center", (c) "Right".

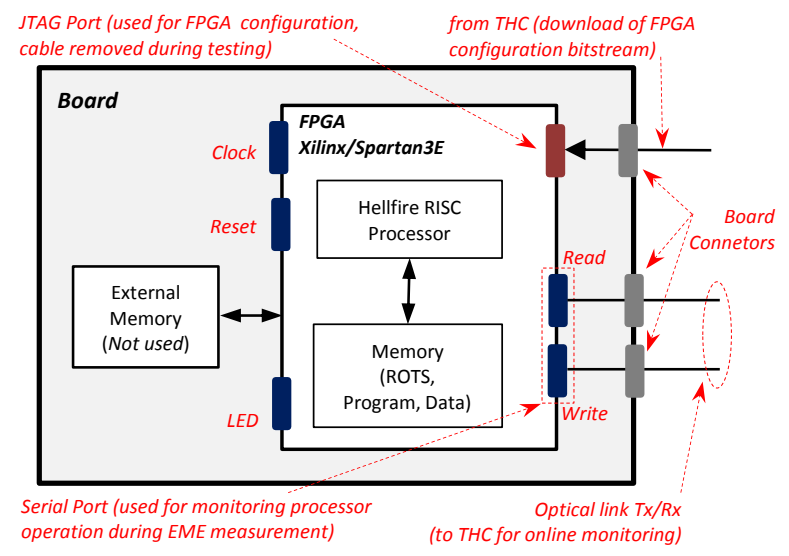

Fig. 5. Overview of the embedded system: FPGA and board.

In order to prevent board-level signals from jeopardizing FPGA EME measurements, the whole system (i.e., processor and memory containing operating system, program and data) was mapped inside the FPGA. The only signals flowing on and outside board during EM emission measurement were those associated to the serial communication connecting the FPGA to the user test host computer (THC). This serial port was used to monitor processor operation in real time during the experiments. In order to prevent communication from being disrupted by electromagnetic interference during the experiments, the serial communication was implemented by means of a two-module optic fiber link (Tx/Rx). Figs. 4 and 5 (five blue dots) depict the pin positions configured around the 
FPGA CLB: "Read" and "Write" (for the serial communication), "LED" (to monitor FPGA operation), "Clock" and "Reset". The pin positions were fixed for all three P\&R configurations. Additionally, a JTAG connection was used to configure the FPGA (Fig. 5, red dot). Once the configuration was complete, the cable was removed before starting EME measurements.

\section{OBTAINED RESULTS}

Fig. 6 summarizes the EME measurements. Fig. 6a depicts results for the LAAS-CNRS experiment, whereas Figs. $6 \mathrm{~b}$ and 6c present results for the two TU Graz experiments. Table I summarizes the ambient noise values measured for the three experiments. The ambient noise was measured by disconnecting the board under test from power supply lines and then, the Spectrum Analyzer captured the remaining noise around the FPGA.

(c)



(b)



(a)

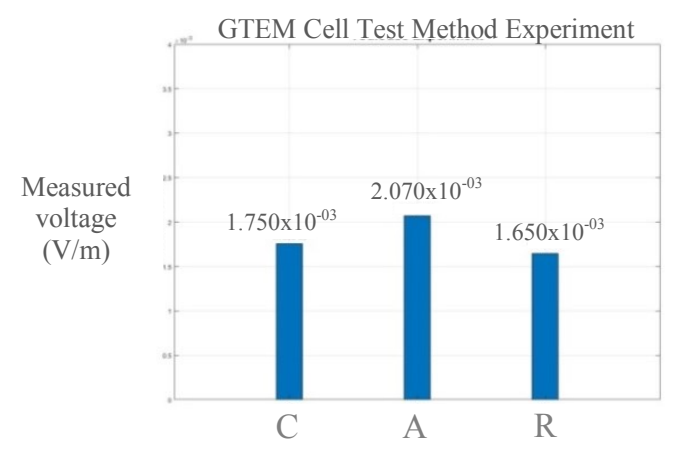

Fig. 6. EME measurements for the three FPGA P\&R strategies (C: "Center", A: "Automated", R: "Right"), performed during the three experiments: (a) GTEM Cell Test Method; (b) Anechoic Chamber with Fixed Near-Field Probe; (c) Surface Scan Method (Moving Near-Field Probe).
By observing Fig. 6 and Table I, the following conclusions can be taken:

a) In Fig. 6a, the emission level of the "Automated" place and route version is $21.8 \%$ higher than the average of the other two configurations ("Centered" and "Right" versions). Note that the GTEM Cell Test Method measures EM emission from the whole system, i.e., not only from the FPGA but also from the board (including tracks and other components such the serial/optical converter logic sitting around the FPGA).

TABLE I. AMBIENT NOISE MEASURED FOR THE THREE EXPERIMENTS

\begin{tabular}{|l|c|}
\hline Experiment & Ambient Noise \\
\hline $\begin{array}{l}\text { Surface Scan (Moving Near-Field Probe) } \\
\text { Method }\end{array}$ & $3.297 \times 10-3(\mathrm{~V})$ \\
\hline $\begin{array}{l}\text { Anechoic Chamber with Fixed Near-Field } \\
\text { Probe }\end{array}$ & $4.766 \times 10-3(\mathrm{~V})$ \\
\hline GTEM Cell Test Method & $8.938 \times 10-4(\mathrm{~V} / \mathrm{m})$ \\
\hline
\end{tabular}

b) In Fig. 6b, the emission level of the three P\&R strategies is similar: $1.649 \times 10^{-2}$ volts. This experiment was not able to identify different emission levels among the threes strategies, as did the other two experiments of Fig. $6 \mathrm{a}$ and $6 \mathrm{c}$. This could be explained by the fact that the measurements in the small anechoic chamber were performed under $44.56 \%$ higher ambient noise than the other near-field technique $\left(4.766 \times 10^{-3}\right.$ volts against $3.297 \times 10^{-3}$ volts, Table I). This high noise level may have jeopardized the results in such a way that EME measured from the FPGA was observed to be in the same order of magnitude for the three P\&R strategies.

c) In Fig. 6c, the EM emission level of the "Automated" place and route version is $8.4 \%$ higher than the other two configurations ("Centered" and "Right" versions).

\section{CONCLUSIONS}

This work presented a preliminary study about the impact of the place and route (P\&R) strategy on the electromagnetic (EM) emission level of a commercial FPGA. The considered device was a Xilinx Spartan 3E, part number XC3S500E4PQ208, which was configured with the Hellfire softcore RISC processor available in the github public domain. The FPGA was mapped by three different strategies: "Automated", in which the processor was placed and routed automatically by the ISE-EDK Design Tool of Xilinx. In the second and third versions, the processor was completely and intentionally placed in the left-hand side ("Right" strategy), and in the periphery region ("Center" strategy) of the configurable logic block (CLB) array of the FPGA. The processor executed a Bubble-Sort program running over an operating system (OS).

The obtained results suggest that the P\&R strategy can affect the FPGA emission level. For the developed experiments, the influence was in the order of $21.8 \%$ (GTEM Cell Test Method) and 8.4\% (Surface Scan Method). The anechoic chamber with fixed near-field probe experiment presented high ambient noise at laboratory, which prevented us from getting measurements with sensitiveness high enough 
to discriminate the emission levels generated by the three P\&R strategies.

\section{ACKNOWLEDGEMENT}

This work has been supported in part by CNPq (National Science Foundation, Brazil) under contract n. 300124/2019-0 (PQ), and Coordenação de Aperfeiçoamento de Pessoal de Nível Superior - Brasil (CAPES) - Finance Code 001.

\section{REFERENCES}

[1] King Lee Chua, Mohd Zarar Mohd Jenu, Chee Seong Fong, See Hour Ying, "Characterizations of FPGA chip emissions based on GTEM cell measurements", 2012 Asia-Pacific Symposium on Electromagnetic Compatibility, DOI: 10.1109/APEMC.2012.6237857.

[2] Van Toan Nguyen, Minh Tung Dam, Jeong-Gun Lee, "Electromagnetic emanation exploration in FPGA-based digital design", Journal Central South University, 26, pp. 158-167 (2019), DOI: https://doi.org/10.1007/s11771-019-3990-1.

[3] Mohamed Ramdani, Etienne Sicard, Alexandre Boyer, Sonia Ben Dhia, James J. Whalen, Todd H. Hubing, Mart Coenen, Osami Wada, "The electromagnetic compatibility of integrated circuits-past, present, and future", Journal IEEE Transaction on Electromagnetic Compatibility, 2009, 51(1): 78-100, DOI: 10.1109/TEMC.2008.2008907.

[4] Jan Mocha, T. Woźnica, "Electromagnetic emission of microprocessors systems implemented in FPGAs" Dec. 2009, Przeglad Elektrotechniczny 86(3):157-160,

www.researchgate.net/publication/291129082_Electromagnetic_emissio n_of_microprocessors_systems_implemented_in_FPGAs

[5] J. Szczęsny, J. F. Kołodziejski, D. Obrębski, "Electromagnetic emission of digital circuits implemented in Xilinx FPGAs XC 4025E and XCV 800", Electron Technology: Internet Journal, 2004 Vol. 36, nr 2 http://yadda.icm.edu.pl/baztech/element/bwmeta1.element.baztecharticle-BWA2-0014-0020.

[6] Juliano Benfica, Letícia B. Poehls; Fabian Vargas, José Lipovetzky, Ariel Lutenberg; Edmundo Gatti; Fernando Hernandez, "Customized Platform for TID and EMI IC Combined Measurements: Case-Study and Experimental Results", Journal of Electronic Testing, v.28, pp. 803-816, Dec.2012, DOI: 10.1007/s10836-012-5334-z.

[7] Juliano Benfica, Bruno Green, Bruno C. Porcher, Letícia Bolzani Poehls, Fabian Vargas, Nilberto H. Medina, Nemitala Added, Vitor A. P. de Aguiar, Eduardo L. A. Macchione, Fernando Aguirre, Marcilei Aparecida G. da Silva, Martin Perez, Miguel Sofo Haro, Ivan Sidelnik, Jeronimo Blostein, Jose Lipovetzky, Eduardo A. Bezerra, "Analysis of SRAM-Based FPGA SEU Sensitivity to Combined EMI and TIDImprinted Effects", IEEE Transactions on Nuclear Science Vol: 63, Issue: 2, 2016, pp. 1294 - 1300, DOI: 10.1109/TNS.2016.2523458.

[8] Alexandra Aguiar, Sergio J. Filho, Felipe G. Magalhaes, Thiago D. Casagrande, Fabiano Hessel, "Hellfire: A design framework for critical embedded systems' applications", $201011^{\text {th }}$ International Symposium on Quality Electronic Design (ISQED), pp. 730-737, 2010, DOI: 10.1109/ISQED.2010.5450495.

[9] https://github.com/sjohann81/hellfireos, last access: March 2020.

[10] IEC 61967-2: 2005, "Integrated circuits - Measurement of electromagnetic emissions, $150 \mathrm{kHz}$ to $1 \mathrm{GHz}$ - Part 2: Measurement of radiated emissions - TEM cell and wideband TEM cell method" TC 47/SC 47A, Ed. 1.0, Int. Electrotechnical Commission, 2005-09-29.

[11] IEC TS 61967-3:2014, "Integrated circuits - Measurement of electromagnetic emissions - Part 3: Measurement of radiated emissions Surface scan method," TC 47/SC 47A, Ed. 2.0, International Electrotechnical Commission, 2014-08-25.

[12] Perry F. Wilson, "On correlating TEM cell and OATS emission measurements", IEEE Transactions on Electromagnetic Compatibility, vol. 37, no. 1, Feb. 1995, pp. 1-16, DOI: 10.1109/15.350235.

[13] Susanne Bauer, Oszkár Bíró, Gergely Koczka, Andreas Gleinser, Gunter Winkler, Bernd Deutschmann, "Time Domain FEM Computational Approach for Calibration of Surface Scan Method", 2018 IEEE International Symposium on Electromagnetic Compatibility and 2018 IEEE Asian-Pacific Symposium on Electromagnetic Compatibility, EMC/APEMC 2018, pp. 866-871, DOI: 10.1109/ISEMC.2018.8393905.
[14] Ronald De Smedt, Steven Criel, Frans Bonjean, Guido Spildooren, Guy Monier, Bernard Demoulin, Jacques Baudet, "TEM cell measurements of an active EMC test chip", IEEE Int. Symposium on Electromagnetic Compatibility, USA, 2000, DOI: 10.1109/ISEMC.2000.874743.

[15] King L. Chua, Mohd Z. M. Jenu, Man_On Wong, See_Hour Ying, "Radiated emissions estimation of an integrated circuit based on measurements in GTEM cell", Proc. Asia-Pacific Symposium on Electromagnetic Compatibility (APEMC), 20-23 May 2013, DOI: 10.1109/APEMC.2013.7360636.

[16] Jin Shi, Michael A. Cracraft, Jianmin Zhang, Richard E. DuBroff, Kevin Slattery, "Using near-field scanning to predict radiated fields", 2004 International Symposium on Electromagnetic Compatibility (IEEE Cat. No.04CH37559), Vol. 1, pp. 14-18, Silicon Valley, CA, USA, 2004, DOI: 10.1109/ISEMC.2004.1349988. 